la poudre, mais c'était pour la boulangerie, la chocolaterie, etc. ; ici, le rendement financier, quoique bon, est cependant assez aléatoire vu la concurrence. S'il devient possible de faire du fromage, la chose apparaît autrement intéressante, car la consommation du fromage va en augmentant, et la demande, l'hiver, est toujours importante et les prix sont soutenus. Mais faisons hautement remarquer aux industriels dont l'intention serait de faire du fromage avec de la poudre de lait, qu'ils aient à orienter leur fabrication de lait desséché dans une direction donnée, qu'iis se gardent bien de dessécher des laits acides, et surtout de les neutraliser au préalable; on obtiendrait des poudres alcalines dont l'emprésurage pourrait conduire à des résultats défectueux. même après l'addition du levain lactique.

La fabrication des fromages en partant de la poudre maigre doit faire appel à une addition de matière grasse, car nous ne songeons nullement ici aux fromages maigres; ce sont des produits meilleurs que nous envisageons.

Sous quelle forme alors la matière grasse sera-t-elle ajoutée au lait maigre reconstitué? Sera-ce du beurre fait de crème douce qu'on émulsionnera eomme il a été vu plus haut? Ou mélangera-t-on en proportions convenables au lait écrémé reconstitué du lait entier frais ou de la crème? Il y a plusieurs modalités à envisager et sur lesquelles la pratique sera à même de nous fixer. 11 importe cependant de relever que si l'on avait recours à du lait entier ou à de la crème, il serait indiqué de les pasteuriser au préalable avant de les ajouter au lait maigre reconstitué, sinon on associerait deux états bactériens différents, celui de la poudre maigre, lequel se traduit par une relative asepsie, et celui du lait entier ou de la crème fraîche, plutôt mal connu.

Nous conclurons en disant que nous n'avons entendu fournic ici que des directions générales, souligner les principales objections, et donner, nous le pensons du moins, le moyen d'y répondre; mais nous nous tromperions fort si l'emploi des bonnes poudres de lait, bonnes au triple point de vue: microbien, physico-chimique et colloïdal, ne prenait pas place un jour dans l'industrie fromagère.

\title{
UN ESSAI DE FABRICATION DE FROIMAGE AVEC DU LAIT SEC
}

par MM. Ch. PORCHER et R. DESRANTES.

Il importe tout d'abord de donner en quelques mots l'histoire de la poudre qui a servi à faire les essais que nous avons tentés, M. DEsRantes et moi l'an dernier.

Il s'agissait d'une poudre maigre fabriquée par le procédé KRAUSE et qui m'avait été adressée par la firme italienne PoLANGHI de Codogno (Lombardie); c'était une poudre fort soluble dans l'eau, très appétissante. 
J'avais fait venir cette poudre en vue de faire des essais de fromagerie et voulant arriver à des résultats aussi concluants que possibles, j'avais pensé bien faire en envoyant le colis, dès son arrivée, dans une Ecole de Fromagerie où on aurait pu tenter avec ce produit diverses fabrications de fromages, pâtes molles, pâtes demi-cuites, pâtes fermes. Malheureusement rien n'a été fait et la poudre m'a été retournée sur ma demande plus de 6 mois après. Elle était encore appétissante, mais, comme son emballage n'était pas étanche - e'était une simple caisse en bois -, la poudre qui, comme on le sait, est fortement hygroscopique, s'était légèrement humidifiée et avait perdu un peu de sa solubilité. Ne voulant cependant pas la jeter, j'ai prié M. Desrantes de faire quelques fromages simples, et ce sont les résultats qu'il a obtenus qu'il veut bien consigner ici.

(Ch. P.)

$$
*^{*} *
$$

La poudre employée, comme il a été dit ci-dessus, avait été fabriquée au moyen du procédé Krause ; elle était vieille de près d'un an. Cette poudre, très fine, donnant sous le doigt le craquelant de la fécule, se "mouillait mal ". Il en est d'ailleurs ainsi de beaucoup de substances pulvérulentes. La poudre quoique soluble, montait à la surface en masses plus ou moins grosses qu'il fallait écraser avec les doigts pour permettre leur dissolution. Malgré son âge, la poudre avait-une odeur qui n'était pas mauvaise.

A l'analyse, elle a donné les résultats suivants :

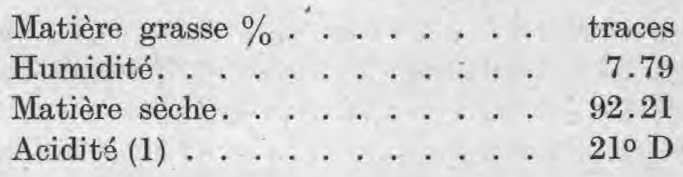

L'essai auquel nous avons procédé a porté sur une fabrication de fromage genre Saint-Marcellin; fromage très connu dans la région lyonnaise.

Reconstitution du lait. - Le lait a été reconstitué de la façon suivante :

$$
\begin{aligned}
& \text { Eau tiède ... . . . . } 72 \text { kg } \\
& \text { Sérum de lait eaillé .... } 17 \text { " } \\
& \text { Lait sec . . . . . . } \frac{11 \text { " }}{100 \mathrm{~kg}}
\end{aligned}
$$

Le sérum provenant de lait caillé a été ajouté voloniairement de façon à apporter d'une part les microbes indispensables à la fermentation et la maturation des fromages et d'autre part, l'acidité nécessaire au milieu pour la bonne réussite de la coagulation du lait reconstitué.

L'acidité du lait ainsi reconstitué était de $34^{\circ}$ DoRnIC. 
En vue de faciliter la coagulation, il a été ajouté 0 gr. 25 de $\mathrm{CaCl}^{2}$ par litre de lait reconstitué (cette dose s'est, par la suite révélée insuffisante. Il aurait fallu très probablement employer I. gr par litre).

Emprésurage. - La mise en présure a eu lieu immédiatement après la reconstitution. La température était alors de $30^{\circ} \mathrm{C}$. Il a été ajouté 1 cc. 5 de présure liquide FABRE de force 2500, par litre de lait reconstitué.

Coagulation. - Le lait a eommencé «à prendre» environ 30 minutes après la mise en présure.

Le lendemain matin, c'est-à-dire après 14 heures, le caillé titrait $80^{\circ}$ DoRnio pour $10 \mathrm{cc}$. La fermentation lactique s'était donc fortement développée.

Cependant le caillé était mou, ne se "tenait " pas et n'a pu être mis en moules que le surlendemain matin.

Au moment du moulage, le caillé était toujours mou, n'avait pas de corps. Il a néanmoins été, avec beaucoup de précautions, mis en moules et on a obtenu 78 fromages.

Retournage et salage. - Le lendemain de la mise en moules, les fromages ont été tournés. Le soir ils ont été changés de moules.

Ces fromages se sont très bien égouttés. La pâte, au toucher, paraissait onctueuse, grasse même.

Cependant le goût n'était pas des plus agréable et très acide. Le salage a eu lieu le $3^{\text {e }}$ jour.

A ce moment-là, les fromages, qui dosaient $78 \%$ d'humidité étaient encore très mous.

Nous résumons ci-dessous les $\mathbf{Z}$ dates des différentes phases de la fabrication :

Emprésurage: 15 mars 1926 (17 h.)

$\begin{array}{llll}\text { Emprésurage : } 15 \text { mars } & 1926 & (17 \mathrm{~h} .) \\ \text { Moulage } & 17 \text { mars } " ~ & (9 \mathrm{~h} .) \\ \text { Retournage } & : 18 \text { mars } ~ & \text { matin } \\ \text { Salage } & : 19 \text { mars } ~ & \text { matin }\end{array}$

Affinage, - Au bout de 12 jours et après des soins normaux, ces fromages se présentaient sous un bel aspect, tant à l'intérieur qu'à l'extérieur.

Les moissisures se sont développées très normalement. Les fromages avaient une belle tenue et donnaient l'impression d'avoir été fabriqués avec du lait non écrémé. Malheureusement ils avaient conservé leur mauvais goût du début et étaient très acides.

Rendement et analyse. - Le poids des 78 fromages fabriqués a été de $7 \mathrm{~kg}$. 750 , soit près de $100 \mathrm{gr}$. par fromage. Le rendement se trouve done être de $7.750 \%$ du lait reconstitué. L'humidité des fromages, qui était de $78 \%$ au moment du salage (le 19 mars) n'était plus que de $62 \%$ le 31 mars. La matière sèche était donc passé de 22 à $38 \%$. 
La perte d'humidité en 12 jours a été ainsi de $16 \%$.

La perte au moulage, par suite du mauvais caillé obtenu a été très forte. En effet, la matière sèche totale des $11 \mathrm{~kg}$. de poudre employée $92,21 \times 11$

étant de $\frac{92,21 \times 11}{100}=10 \mathrm{~kg}, 143$ et le poids total de la matière sèche 100

contenue dans les 78 fromages étant de $\frac{7.750 \times 38}{100}=2 \mathrm{~kg} .945$,

il y a donc eu perte de $10.143-2,945=7 \mathrm{~kg}$. 198 de matière sèche.

Cette perte provient du fait que le caillé, étant trop mou, s'est échappé par les trous des moules, entrainé par le petit-lait d'égouttage.

Cet essai, malgré la perte formidable de caillé qui s'est produite malgré tout très intéressant même, au point de vue économique.

En effet, si nous comptons le lait en poudre écrémé à raison de $4 \mathrm{fr}$. le $\mathrm{kg}$ et le prix de vente des Saint-Marcellin à $8 \mathrm{fr}$. le $\mathrm{kg}$. nous obtenons en adoptant les chiffres de cet essai :

$$
\begin{gathered}
\text { Valeur de } 11 \mathrm{~kg} \text {. de poudre : } 4 \times 11= \\
\text { Valeur de } 7 \mathrm{~kg} \text {. } 750 \text { de fromages à } 8 \mathrm{fr} . \\
\text { Bénéfice brut }
\end{gathered}
$$

La seule difficulté réside actuellement dans la coagulation du lait reconstitué. Le jour où sa coagulation méthodique sera résolue, les plus grands espoirs seront permis et la portée économique de cette nouvelle industrie incalculable.

$$
*^{*} *
$$

Ces essais seront continués avec des poudres plus fraîches, bien conditionnées et qui n'auront pas trainé dans des pièces plus ou moins humides, comme l'avait fait le lait sec qui a servi aux essais ci-dessus.

Nous serons heureux de tenir les lecteurs de la Revue Le Lait au courant de nos essais ultérieurs.

\title{
LES EAUX RÉSIDUAIRES DES LAITERIES
}

\author{
par Paul ROSSI, \\ Directeur des Services Vétérinaires de Saône-et-Lolre
}

L'extension rapide et imprévue de l'industrie laitière a fait apparaître, dans toutes les régions de France, un lourd problème dont la solution, encore incomplète, ne pourra devenir définitive qu'après de nouvelles et patientes recherches; c'est celui de l'évacuation des eaux résiduaires.

Ce problème très complexe, dont les conséquences pour l'hygiène sont graves, n'a attiré l'attention des hygiénistes qu'à la suite de sérieux 